\title{
Hemangiosarcoma of the adrenal glands: CT findings in two cases
}

\author{
F. Ferrozzi, G. Tognini, D. Bova,* G. Zuccoli, P. Pavone \\ Istituto di Scienze Radiologiche, Università degli Studi di Parma, Viale Gramsci, 14, Parma, Italy I-43100
}

Received: 18 November 1999/Accepted after revision: 14 August 2000

\begin{abstract}
Hemangiosarcomas of the adrenal gland, both benign and malignant, are exceedingly rare; only a dozen of them have been reported in literature to our knowledge. We describe herein the findings at CT of 2 primary hemangiosarcomas of the adrenal glands. We also discuss their etiology, incidence, and differential diagnosis, particularly in relation to more frequent primary adrenal lesions, such as adrenal adenomas and carcinomas. CT will generally show a heterogeneous mass with frequent necrosis, contrast-enhancement, and occasional calcifications. A prospective diagnosis cannot be made by imaging alone, and pathology represents the discriminating method for a correct diagnosis.
\end{abstract}

Key words: Adrenal gland, neoplasms-Adrenal gland, computed tomography.

Primary malignant neoplasms of the adrenal gland are uncommon and may involve the adrenal cortex (adenocarcinoma) or the adrenal medulla (pheochromocytoma and neuroblastoma) $[1,2]$. The adenocarcinoma is the most frequent primary malignant tumor of the adrenal gland. Vascular tumors of the adrenal gland, both benign and malignant, are exceedingly rare. The angiosarcoma probably represents approximately $1-2 \%$ of these tumors.

Angiosarcomas occur in the skin, breast, retroperitoneum, and visceral organs, with the liver being the most frequent site of visceral organ involvement [3]. Only a dozen primary angiosarcomas of the adrenal gland have been reported in the literature to our knowledge [1, 4-8], the first case being described in 1988 by Kareti et al.

* Present address: Department of Radiology, Loyola University Medical Center, 2160 South First Avenue, Maywood, IL 60153, USA

Correspondence to: D. Bova

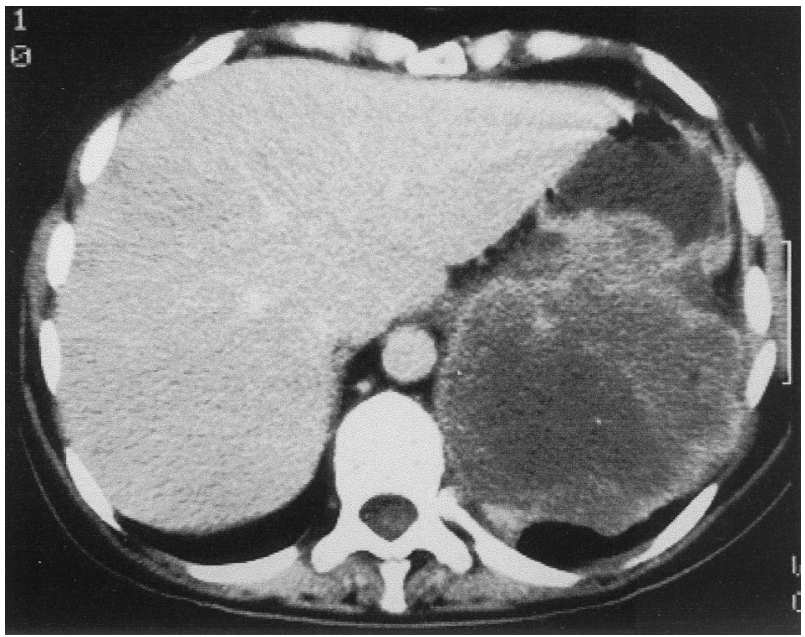

Fig. 1. CT of the abdomen during intravenous contrast medium administration demonstrates a voluminous, predominately necrotic, mass in the left retroperitoneum, with a thin and irregular rim of peripheral enhancement.

Metastatic involvement of the adrenals by angiosarcomas is much more frequent.

We describe the computed tomographic (CT) findings of two primary hemangiosarcomas of the adrenal glands. We also discuss their etiology, incidence, and differential diagnosis, particularly in relation to more frequent primary adrenal lesions, such as adrenal adenomas and carcinomas.

\section{Case reports}

\section{Case 1}

A 67-year-old male, without a significant medical history, presented with left flank pain of 6 months' duration. The patient exhibited no hematuria or hypertension. An intra- 


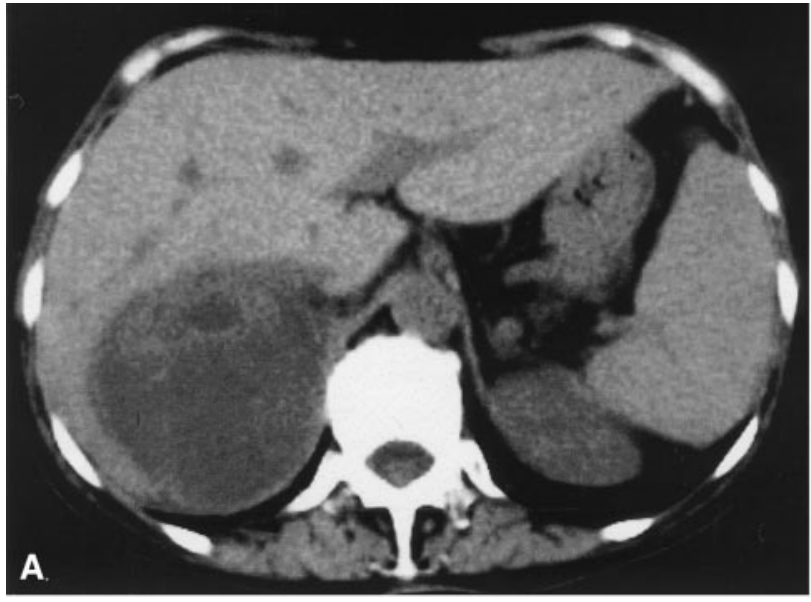

Fig. 2. A CT of the abdomen demonstrates a large, heterogeneous, hypodense mass of the right retroperitoneum, with irregular septations, compressing the hepatic parenchyma. B After intravenous contrast administration, enhancement is visualized along a thin peripheral rim and

venous pyelogram showed a left suprarenal mass, measuring approximately $13 \mathrm{~cm}$ in diameter, displacing the kidney inferiorly and the spleen superiorly. CT of the abdomen showed an expansile process of the retroperitoneum, in the left adrenal fossa, with an infiltrating pattern of growth and extensive necrotic changes centrally (Fig. 1). Urinary catecholamines, vanillylmandelic acid, and corticosteroid metabolites were all within the normal limits.

Through a combined thoraco-abdominal approach, a radical dissection of the mass, after isolation from the kidney, spleen, and pancreatic tail, was performed. At pathology, the 810-g mass showed a fluid center and a peripheral rim of solid spongy tissue, with hypervascular nodules. At microscopy, communicating vascular spaces of variable dimensions, lined by epithelioid cells, with interposed connective columns, were found. Immunohistochemical analysis was positive for factor VIII in about $20 \%$ of neoplastic cells, thus confirming the endothelial nature of the neoplasm, which was finally described as an epithelioid hemangiosarcoma. Retrospective analysis of the patient's history did not reveal risk factors such as familial angiodysplasia, prior radiation therapy, or exposure to toxic chemicals.

The patient expired 2 months later with diffuse metastatization and pleural hemorrhagic phenomena secondary to metastases.

\section{Case 2}

A 60-year-old female presented to an outside institution with acute and sharp right upper quadrant pain. Her medical history was significant for peptic ulcer disease, a thyroid cyst removed at age 53, a total hysterectomy and

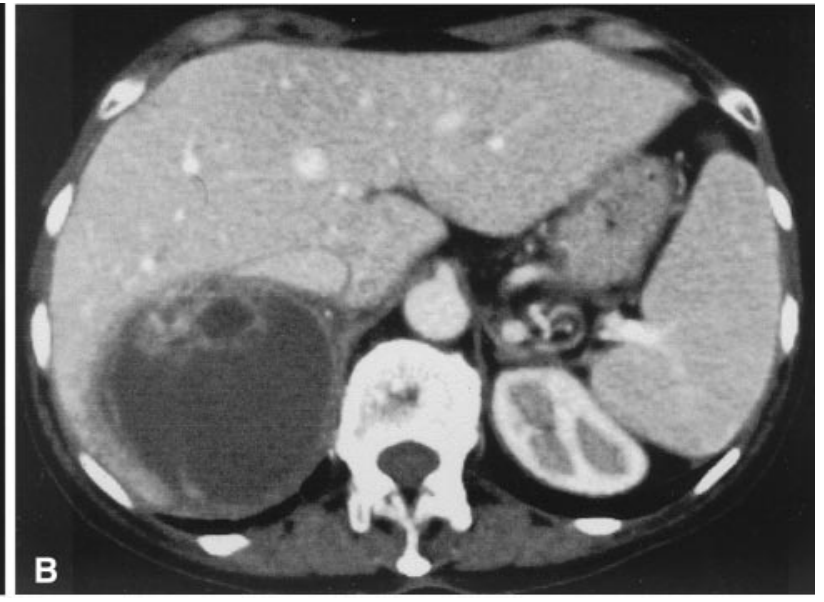

in the irregular anterior septa. The predominately hypodense component shows no increase in density and is therefore significant for extensive necrosis.

salpingo-oophorectomy for adenocarcinoma of the uterine cervix with subsequent radiation therapy at age 56, acute small bowel obstruction with surgical lysis of adhesions at age 57, and laparoscopic cholecystectomy 2 months prior to this hospitalization.

Emergent ultrasonography showed an oval mass in the right retroperitoneum measuring $7 \times 5 \times 4 \mathrm{~cm}$, with heterogeneous echotexture, small anechoic areas, and punctate calcifications, suggestive of adrenal hematoma. CT without contrast medium administration confirmed a mass with heterogeneous attenuation, with an appearance suggestive of an organizing hematoma. At laparotomy, the hemorrhagic collection was drained and sent to pathology, where fibroadipose tissue with an organizing hematoma was diagnosed. The patient had persistent lumbar and right upper quadrant pain at discharge, for which she was referred to our institution. CT before and after contrast medium administration (Fig. 2) demonstrated a voluminous mass of the right adrenal fossa, measuring approximately $10 \times 9 \mathrm{~cm}$. The mass displayed a large fluid central component with a peripheral, thin, irregular rim of vascular solid tissue. The lesion appeared to indent the right hepatic lobe and displace the right kidney inferiorly and laterally. The liver was free of focal abnormalities.

At surgery, an $11-\mathrm{cm}$, right adrenal mass, adhering to the liver and the diaphragm, was isolated and dissected out. The normal adrenal tissue was compressed along the superior aspect of the mass. At pathology, the neoplastic structure appeared to be constituted by anastomosed vascular spaces with endothelial cells and papillary stroma. Immunohistochemical analysis was positive for factor VIII in virtually all cells. The mass was diagnosed as a hemangiosarcoma. 


\section{Discussion}

Hemangiosarcoma is a rare mesenchymal tumor of the adrenal gland, usually associated with a poor prognosis. There is little information about its distribution, but it probably occurs in the same age group as other hemangiosarcomas (sixth and seventh decades of life) [6]. The most widely recognized predisposing factors in angiosarcoma involving other areas of the body include chronic lymphedema, anabolic steroid therapy, thorotrast, and familial angiodysplasia. Occupational or environmental carcinogens such as vinyl chloride, arsenic, and copper have also been linked to the development of angiosarcoma [8]. The first correlation between chronic exposure to arsenic compounds and hemangiosarcomas was proposed in 1957, but the carcinogenic effect of arsenic on the adrenals was not reported until 1991, when Livaditou et al. presented a case of epithelioid adrenal hemangiosarcoma, probably secondary to use of arsenic-containing insecticides [6]. In 1992, Ofer Ben-Izhak et al. presented a case report of epithelioid adrenal hemangiosarcoma with associated mesenteric fibromatosis and an elevated serum level of estradiol [5].

Hemangiosarcoma of the adrenals has no functional activity; its clinical presentation is nonspecific and is characterized by an abdominal mass with or without associated flank pain, weight loss, fever, and weakness. These tumors are of variable size at presentation. The normal adrenal tissue is generally displaced and compressed toward the periphery. The adrenal mass demonstrates extensive central necrosis and hemorrhagic infiltration. Its microscopic appearance is that of anastomosed vascular spaces of different sizes and shapes, with papillae of stroma and neoplastic endothelial cells [1]. Ultrastructural studies have confirmed the endothelial origin of the tumoral cells [9]. Immunohistochemistry provides ultimate evidence of the vascular origin of tumors otherwise nonspecific in appearance. At pathology, adrenal epithelioid hemangiosarcoma generally appear as large masses with central necrosis and hemorrhagic infiltration.

The appearance of adrenal hemangiosarcomas on the various imaging modalities is nonspecific. Nonenhanced CT usually shows a heterogeneous suprarenal mass with necrotic changes, massive liquefaction, and peripheral contrast enhancement. Calcifications are less frequently detected than in carcinomas, and they have a more regular shape, likely representing calcified vascular thrombi. In contrast, calcifications in carcinomas are irregular and dystrophic in origin. On magnetic resonance imaging, the tumor appears as a mass with mildly heterogeneous low signal on T1-weighted images and marked heterogeneity and high signal on T2-weighted images.

Many neoplasms may be confused with the hemangiosarcoma. Adrenal cortical carcinoma is the most important differential diagnosis to consider, but other neoplasms such as pheochromocytoma, metastatic malignancies, retroperitoneal mesenchymal tumors, and adrenal adenomas undergoing massive hemorrhage [10] may simulate the appearance of an angiosarcoma. Differentiation between these lesions cannot be obtained by imaging alone; resection or biopsy must be performed to reach the definitive diagnosis [11]. It is very important to correlate radiologic and pathologic findings. Adrenal cortical carcinoma is a malignant neoplasm originating from adrenal cortical cells, which may or may not have functional activity. Its clinical presentation is very heterogeneous, varying from the classical Cushing's or virilization syndrome to simple biochemical abnormalities associated with tumors forming precursor steroids without hormonal activity. They are often (87\%) unilateral masses [12-14], quite heterogeneous in appearance, with areas of necrosis, hemorrhage, irregular distrophic calcification, and irregular and mild contrast enhancement. Calcifications are less frequent in hemangiosarcoma and have a phlebolithic origin. Adrenal adenomas are relatively common in adults and have been reported to have an incidence of 2-9\% in autopsy series [15, 16]. Adrenal adenomas are almost always smaller than $5 \mathrm{~cm}$, are homogeneously hypodense, almost never show fluid components, and rarely demonstrate calcification. They seldom hemorrhage, with subsequent formation of cystic areas and fibrosis [10], with sudden increase in size, foci of fluid density, calcification, fibrosis, and patchy heterogeneous contrast enhancement.

The organ of origin of a bulky retroperitoneal lesion may be difficult to determine. A retroperitoneal leiomyosarcoma may simulate the appearance of an adrenal hemangiosarcoma. Metastases to the adrenal glands also have a wide range of sizes and appearance, with the larger ones being more heterogeneous. Pheochromocytoma may simulate an angiosarcoma because of its large size, cystic regions, and occasional calcifications. Most pheochromocytomas, however, produce endocrine abnormalities and characteristic symptoms that generally permit a specific diagnosis. Its imaging characteristics on T2-weighted magnetic resonance images and on scintigrams obtained with metaiodobenzylguanidine $[11,16,17]$ are also helpful.

\section{References}

1. Kareti LR, Katlein S, Siew S, et al. Angiosarcoma of the adrenal gland. Arch Pathol Lab Med 1988;112:1163-1165

2. Fiordelise S, Zangrandi A, Tronci A, et al. Angiosarcoma of the adrenal gland. Case report. Arch Ital Urol Nefrol Androl 1992;64: 341-343

3. Bosco PJ, Silverman ML, Zinman LM. Primary angiosarcoma of adrenal gland presenting as paraneoplastic syndrome: case report. J Urol 1991;146:1101-1103

4. Ansari SJ, Ordonez NG, Romsdahl MM, et al. Primary angio- 
sarcoma mimicking adrenal pseudocyst. Am J Surg Pathol 1999; 23:1455-1463

5. Ben-Izhak O, Auslander L, Lichtig C, et al. Epithelioid angiosarcoma of the adrenal gland with cytokeratin expression. Report of a case with accompanying mesenteric fibromatosis. Cancer 1992;69: 1808-1812

6. Livaditou A, Alexiou G, Floros D, et al. Epitheliod angiosarcoma of the adrenal gland associated with chronic arsenical intoxication? Pathol Res Pract 1991;187:284-289

7. Nakagawa N, Takahashi M, Maeda K, et al. Case report: adrenal haemangioma coexinting with malignant haemangioendothelioma. Clin Radiol 1986;37:97-99

8. Wenig BM, Abbondanzo SL, Heffess CS. Epitheliod angiosarcoma of the adrenal glands. A clinicopathologic study of nine cases with a discussion of the implications of finding "epithelial-specific" markers. Am J Surg Pathol 1994;18:62-73

9. Popper H, Thomas TB, Tellers NC, et al. Development of hepatic angiosarcoma in man induced by vinyl chloride, thorotrast and arsenic. Comparison with cases of unknown etiology. Am J Pathol 1978;92:349-369

10. Van Haelst UJGM, Pruszczynski M, Ten Cate LN. Ultrastructural and immunohistochemical study of epithelioid hemangioendothelioma of bone: coexpression of epithelial and endothelial markers. Ultrastruct Pathol 1990;14:141-149

11. Granger JK, Houn HY, Collins C. Massive hemorrhagic functional adrenal adenoma histologically mimicking angiosarcoma: report of a case with immunoistochemical study. Am J Surg Pathol 1991;15: 699-704

12. Newhouse JH, Heffess CS, Wagner BJ, et al. Large degenerated adrenal adenomas: radiologic-pathologic correlation. Radiology 1999;210:385-391

13. D’Agata R, Malozowsky S, Barkan A, et al. Steroid biosyntesis in human adrenal tumors. Horm Metabol Res 1987;19:386-388

14. Sullivan M, Boileau M, Hodges CV. Adrenal cortical carcinoma. J Urol 1978;120:660-665

15. Nader S, Hickey RC, Sellin RV, et al. Adrenal cortical carcinoma. A study of 77 cases. Cancer 1983;52:707-711

16. Bush WH, Elder JS, Crane RE, et al. Cystic pheochromocytoma. Urology 1985;25:332-334

17. Krebs TL, Wagner BL, Kenney PJ. MR appearance of pheochromocytoma of the adrenal gland with pathologic correlation [abstract]. Radiology 1997;205(P):342 